\title{
Conceptos sensibilizantes en la historia de un caso: una reflexión profesional
}

\section{Professional Sensitizing concepts in the history of a case: a professional reflection}

\author{
BEATRIZ MARTÍNEZ PÉREZ \\ Profesora Asociada de la Facultad de Trabajo Social. Universidad de Murcia (España) \\ beatrizmz@um.es.https://orcid.org/0000-0002-8287-8161
}

\begin{abstract}
Resumen: El artículo tiene como objetivos, analizar la Historia Social de un caso de persona mayor y su cuidadora en situación de desprotección, así como las actuaciones profesionales realizadas, y desde ahí se desprende el segundo objetivo, dos conceptos sensibilizantes: evidenciar que toda intervención social implica un tiempo diferencial entre el tiempo Kronos, objetivo de la intervención realizada según protocolo, y el tiempo Kairós, que el sujeto de intervención necesita en su proceso subjetivo de cambio, y cómo esto conduce a reflexionar y comprender que en la acción social, toda actuación debe ser siempre revisada y repensada. La metodología que se utiliza se sustenta en una perspectiva hermenéutica bajo el método cualitativo, donde se utiliza la Historia Social de un caso como instrumento que captura la experiencia de los sujetos intervinientes, viendo los hechos desde la perspectiva de las personas, quienes están continuamente interpretándose y definiéndose en diferentes situaciones. Como conclusiones, se destaca la necesidad de que los sujetos profesionales debemos mantener siempre abierta la revisión, reflexión y la dialéctica de lo que es el trabajo social, el ser y quehacer, no funciona la idea romántica de agente de cambio, si esta implica la pérdida de la claridad de las subjetividades implicadas, que son las que realmente permiten contribuir. Sin embargo la praxis no se puede encorsetar, sírvanos la Historia Social de un caso para revisarnos y reflexionar con el tiempo Kairós al timón. Es el sujeto quien marca el tiempo de la intervención y quien da la mirada de por dónde seguir.
\end{abstract}

Palabras clave: historial social, khronos, kairós, reflexión, praxis profesional.

\begin{abstract}
Summary: the aim of this article is to analyze the social history of a case of the elderly and their caregiver in a situation of unprotection as well as the professional actions carried out and from there the second objective emerges, two sensitizing concepts: evince that all social intervention implies a differential time between the objective Kronos time of the intervention carried out according to protocol and the Kairos time that the intervention subject needs in its subjective process of change and how this leads to reflect and understand that in the whole social action performance must always be reviewed and rethought. The methodology used is based on a hermeneutical perspective under the qualitative method where the social history of a case is used as an instrument that captures the experience of the intervening subjects, seeing the facts from the perspective of the people, who are continuously
\end{abstract}


interpreting and defining in different situations. In conclusion, is highlight the need for professional subjects that should always keep open the review, reflection and dialectic of what social work, being and work are, the romantic idea of a change agent does not work if it implies loss from the clarity of the implied subjectivities that are the ones that can really contribute to the change, the praxis cannot be corseted, serve us the social history of a case to review and reflect with the time Kairos at the helm, it is the subject who marks the time of the intervention and who gives the look of where to continue.

Keywords: social history, khronos, kairós, reflection, professional praxis.

\section{INTRODUCCIÓN}

El presente artículo se sustenta principalmente sobre la descripción detallada de la historia social de un caso de persona mayor y su cuidadora, ambas en situación de desprotección como argumento privilegiado a través del cual poder observar, por un lado los datos relevantes y significativos durante el proceso de acompañamiento y apoyo personalizado, así como la vivencia subjetiva de los sujetos protagonistas en su propio proceso durante la intervención; y por otro la praxis profesional desarrollada durante la intervención social. El análisis de la historia social del caso como instrumento de investigación presenta dos objetivos: evidenciar que toda intervención social implica un tiempo diferencial entre el tiempo kronos, objetivo de la intervención realizada según protocolo, y el tiempo kairós, que el sujeto de intervención necesita en su proceso de vivencia al cambio y cómo esto lleva a reflexionar sobre las historias de casos, las historias de vida constituyen una materia privilegiada para comprender que en la acción social toda actuación debe ser siempre revisada y repensada.

La historia social del caso se presenta de manera organizada cronológicamente, recogiendo fielmente los hechos y el diagnóstico profesional, y esto expuesto en cada fase o etapa de la intervención: inicio y antecedentes, desarrollo de las actuaciones realizadas siguiendo los protocolos existentes, la praxis profesional experimentada y la hegemonía de la institución, y finalmente el desenlace de la intervención. Se pretende llevar al lector hacia los objetivos del artículo arriba mencionados, así como también al recurso del espíritu crítico y responsable del trabajo social como ciencia aplicada y por ende en continua revisión.

La revisión fenomenológica de este caso invita a la reflexión en el maremágnum de actuaciones acometidas por los/as profesionales con el tiempo khronos como timón, en la urgencia de llegar a la meta legítima de protección de los sujetos más desprotegidos, obviando la necesidad subjetiva del sujeto de la intervención que implica un tiempo kairós.
Es necesario hacer una lectura analítica y autorreflexiva, poniendo la intención en la observación de la vivencia y el comportamiento de los sujetos implicados objeto de la intervención, y las actuaciones y medidas de protección emprendidas, que dan sentido y justifican la buena práctica profesional bajo las cristalizadas instituciones, así como, el desenlace real de la Historia Social del caso, pues hacen emerger algunos conceptos sensibilizantes (Ferrarotti, 2007) que indican la dirección en la cual se necesita excavar y explorar. La Historia de un caso induce al profesional a practicar la virtud de la humildad, por tanto se expone a continuación la Historia Social de un caso registrada como material exclusivo para ello.

Para el presente trabajo no hay reglas metodológicas establecidas o que constituyan en sentido estricto los objetivos plateados, pues la propia descripción y proceso de la Historia Social del caso dota de comprensión la necesidad de reflexionar y revisar el claroscuro de la práctica profesional.

\section{METODOLOGÍA}

El proceso de investigación del presente trabajo se sustenta en una perspectiva hermenéutica de revisión y comprensión bajo una metodología cualitativa, donde la historia social de un caso conforma una perspectiva fenomenológica, la cual visualiza la conducta humana, lo que las personas dicen y hacen, como el producto de la definición de su mundo. Algunos autores señalan que la perspectiva fenomenológica representa un enfoque medular en el entramado de la metodología cualitativa (Ferrarotti, 2007).

En este sentido, la historia social de un caso, como instrumento de metodología cualitativa, busca capturar tal proceso de interpretación comprendiendo la experiencia de los sujetos intervinientes, viendo las cosas desde la perspectiva de las personas, quienes están continuamente interpretándose y definiéndose en diferentes situaciones (Taylor y Bogdan, 2000). Por lo tanto, la metodología cualitativa aquí empleada permite analizar la realidad de la Historia social del caso desde una perspectiva humanista, ya que hunde sus raíces en 
los fundamentos de la fenomenología, el existencialismo y la hermenéutica, tratando de comprender la conducta humana desde el propio marco de referencia de las personas. Facilitando de manera privilegiada la sustracción de conceptos sensibilizantes, que emergen de la realidad de la historia y que conducen al acto humilde de la revisión del quehacer profesional, durante el desarrollo de la intervención en el caso de una persona mayor y su cuidadora, ambas en situación de desprotección.

El desarrollo metodológico del trabajo se basa en dos momentos: 1) la descripción detallada de la historia social del caso desde sus antecedentes, protagonistas, relaciones familiares, factores de riesgo, demanda, actuaciones realizadas, recursos aplicados y desenlace; y 2) la observación y posterior análisis de la historia social de donde se puede sustraer dos conceptos que llamaremos sensibilizantes: a) que existe un tiempo khronos que marca la intervención en el quehacer profesional dentro de una institución, el cual trascurre desacompasado con el tiempo kairós indeterminado, que los sujetos necesitan para que suceda algo que les lleve al cambio, b) finalmente llegar al desenlace del caso que conducirá afortunadamente a la revisión y reflexión de la práctica profesional como ejercicio de humildad.

El método cualitativo, utilizando la técnica de la historia social de un caso, ofrece pues elementos que favorecen a una interpretación más profunda de análisis del quehacer profesional, proporciona la oportunidad de hacer una autorrevisión, pues aporta argumentos concretos y específicos, no solo de los sujetos de intervención sino del profesional y su actuación bajo el techo de la institución, por lo que constituye un argumento de interés para la investigación social o propuestas para una investigación más profunda.

Al mismo tiempo se señala que para el presente artículo y descripción de la historia social del caso han sido alteradas y modificadas las identidades tanto de los profesionales como de los sujetos de intervención. Utilizando nombres y referencias ficticias que preservan el anonimato. En este sentido ningún parecido coincide con la realidad identitaria. Además de contar con la autorización de los participantes para la presente investigación de manera expresa.

\section{NARRATIVA DEL CASO: LA HISTORIA SOCIAL}

A continuación se va a describir la Historia Social del caso cronológicamente a modo de narrativa, con la intención de invitar al lector a ensimismarse en la lectura cual ensayo, de manera que quede a un lado la rigidez de la profesionalidad y la hegemónica praxis bajo el paraguas de la institución, induciendo hacia una lectura que permita conectar con la subjetividad de los sujetos protagonistas de la intervención como también con el hilo de actuaciones de una buena práctica profesional. Es por ello que la Historia Social no se plasmará siguiendo el esquema del método de trabajo social exhaustivo: estudio-diagnóstico, planificación, ejecución y evaluación. Sino que se reflejan los datos más relevantes y significativos durante el proceso de acompañamiento, evitando todos aquellos que no aporten valor a la intervención y manteniendo en todo momento la historia actualizada. Recogiendo fielmente los hechos y el diagnóstico social de los y de la profesional.

Rosa', es una persona mayor de 78 años casada con Alfonso de 81 años de edad. Rosa se ha dedicado a lo largo de su vida a la crianza y tareas del hogar, y Alfonso ha realizado su actividad laboral como trabajador autónomo en la realización de mudanzas mobiliarias. El matrimonio afincado en Totana tuvo ocho hijos, viven siete, los cuales realizan vidas independientes y conforman sus propias unidades familiares a excepción de dos: Paco que es soltero, no tiene hijos, se encuentra en situación de desempleo, presenta una minusvalía del $65 \%$ por discapacidad intelectual y problemas de alcoholismo, Paco vive solo en la casa de campo familiar y Dori también soltera, sin hijos, pensionista, con diagnóstico de enfermedad mental esquizofrenia obsesiva. Dori vive en el domicilio familiar donde es atendida por su madre.

El matrimonio convive durante más de treinta años, sin embargo en el año 2016, a raíz de un episodio de violencia doméstica, Rosa se atreve a interponer denuncia. El Juzgado de Violencia establece orden de alejamiento. Alfonso sale del domicilio trasladándose a vivir a la casa de campo familiar junto a su hijo Paco. Aunque poco tiempo después Rosa retira la denuncia y la orden de alejamiento deja de tener vigencia, el matrimonio no retoma la convivencia. Rosa y su hija Dori continúan en el domicilio familiar.

A partir del episodio de violencia, Rosa, que ya presentaba diagnóstico de la enfermedad de Parkinson, sufre una evolución cabalgante de la enfermedad, precisando de atención y cuidados continuados por una pérdida de autonomía severa. A partir de ese momento es Dori, la hija con problemas de salud mental, quien ejerce las funciones de cuidadora principal de su madre, produciéndose una inversión de roles.

Es importante reflejar la dinámica socio familiar, ya que las relaciones entre los miembros de la unidad familiar influyen notoriamente en el desencadenante de la situación de crisis y desprotección de las dos miembros convivientes: Rosa

1 El nombre de los sujetos de la intervención y datos identificativos han sido modificados para preservar el anonimato. 
y Dori, madre e hijas ambas con limitaciones para realizar las actividades básicas de su vida, lo que las lleva a encontrarse en una situación de dependencia.

Las relaciones paterno-materno-filiares hacia ambos progenitores por parte de los hijos son distantes, esporádicas, con muestras de desentendimiento, con la excepción de las dos hijas menores: Dori y Miriam. Esta última acude diariamente al domicilio familiar para dejar a su hijo de dos años al cuidado también de Dori mientras ella asiste al trabajo. Las relaciones filiares son inexistentes a excepción de las dos hermanas menores que se ven diariamente y cuya relación es conflictiva.

A nivel económico, laboral y ocupacional se destaca que la unidad de convivencia formada por Rosa y Dori cuenta con los ingresos de las pensiones no contributivas de ambas. Los gastos de vivienda, suministros, impuestos, etc. están cubiertos por Alfonso, por lo que la economía no supone un agravamiento de la situación de vulnerabilidad.

A nivel sanitario se describe que Rosa está afectada de: Parkinson, movilidad reducida, obesidad mórbida e incontinencia de esfínteres. Dori está afectada de: esquizofrenia obsesiva, movilidad reducida, obesidad mórbida, deterioro cognitivo progresivo y fobia a salir y relacionarse con el exterior; apenas sale del domicilio evitando con ello ser vista, siente vergüenza de su aspecto físico.

A principios de 2016 la trabajadora social del Centro de Salud pone en conocimiento de los Servicios Sociales de Atención Primaria, la alerta sobre la situación de una persona mayor que presenta limitación severa en la autonomía y que es atendida por un familiar que a su vez precisa de cuidados, así como también de un sobrino menor de dos años.

A partir de ese momento de detección y derivación del caso por parte de los profesionales sanitarios, se establecen las primeras actuaciones de intervención en el caso de manera conjunta y coordinada entre los profesionales de lo social de ambas instituciones, siguiendo los protocolos de actuación.

\section{ACTUACIONES EN LA INTERVENCIÓN SOCIAL DEL CASO}

Seguidamente se describe la secuencia de actuaciones realizadas por los Servicios Sociales de Atención Primaria municipales desde la derivación por parte del Centro de Salud de referencia, así como también las actuaciones conjuntas con otros profesionales, en su mayoría trabajadoras sociales, aunque también otros profesionales del ámbito de lo social hasta la finalización de la intervención.
Tras la detección y derivación del caso por el Centro de Salud, desde Servicios Sociales se comienza la intervención social acometiendo una fase exploratoria que ayude a elaborar un plan de intervención. Fue clave y de vital importancia contar desde el primer momento con el diagnóstico médico tanto de Rosa como de Dori, lo cual permitió pasar a actuaciones concretas que disminuyeran los factores de riesgo que emergían de la situación de una persona mayor con necesidad de cuidados, los cuales estaban siendo ejercidos por una cuidadora de autonomía limitada y susceptible de ser atendida a su vez.

Se realiza una primera visita domiciliaria conjunta entre las trabajadoras sociales de Servicios Sociales: unidad de trabajo social y dependencia y la trabajadora social del Centro de Salud, momento en el que se recoge la demanda explícita de Rosa: La necesidad de contar con un servicio de apoyo que cubra las tareas de limpieza de la vivienda y aseo personal de su persona, pues "mi hija no puede con todo". En relación a los apoyos familiares refiere: "Mis hijos están ocupados y no pueden hacerse cargo" (Ent 1.)

Rosa no hace referencia a la separación del matrimonio por violencia de género ni tampoco a la historia familiar, omitiendo información específica.

Esta primera etapa exploratoria ya permite trazar un plan de intervención que justifica la necesidad de apoyo a través del Programa de servicio de ayuda a domicilio municipal con dos objetivos prioritarios: 1) cubrir las tareas de atención personal de Rosa y 2) descargar a Dori de las tareas como cuidadora principal (Navarro Ramos, 2016).

El caso es derivado desde la unidad de trabajo socia al área especializada de Mayores - Servicio de ayuda a domicilio. Una vez canalizado, la intervención continúa desde el programa específico. Se realiza visita domiciliaria para dar de alta el servicio de ayuda a domicilio con carácter de urgencia, así como la presentación de la auxiliar de hogar de referencia que acudirá dos días a la semana al domicilio. Una vez pasadas unas semanas se realizan visitas de manera periódica para seguimiento del caso, valoración de posibles modificaciones en las tareas y horarios, que de una manera más perfilada supongan una descarga para la cuidadora y una mejora de las rutinas en las actividades básicas de la vida diaria de ambas, así como también se empieza a trabajar en esta ocasión con Dori sobre un tercer objetivo: Solicitar un Centro de Día específico para personas dependientes con problemas de salud mental donde Dori pueda adquirir hábitos, rutinas, establecer relaciones sociales y realizar actividades de ocio que le supongan una mejora en su calidad de vida. Ante dicho planteamiento no se obtiene una respuesta clara o definitiva por parte de Dori, ni acepta ni rechaza el recurso. 
Durante la visita a domicilio de seguimiento en el segundo mes de servicio, Rosa realiza con plenas facultades cognitivas la demanda explícita de ingresar en un centro residencial. Refiriendo:

"Tengo miedo de un posible brote de mi hija, ya que no toma la medicación adecuadamente, duerme a deshoras y cuando le demando atenciones de manera reiterada se torna agresiva. Esta solicitud no es urgente, no quiero ingresar inmediatamente, que se inicien los trámites y sigan su curso" (Ent 3.)

A partir de dicha demanda se inician los trámites de reconocimiento de grado de dependencia. El organismo competente Instituto Murciano de Acción Social resuelve con un grado I, grado que no contempla la concesión de plaza residencial por lo que siguiendo la voluntariedad de Rosa se tramita una segunda solicitud de revisión de grado por agravamiento, dada la situación de vulnerabilidad que presentan ambas convivientes. Rosa ahora a través del reconocimiento de persona dependiente grado I es beneficiaria del servicio de ayuda a domicilio por dependencia. Esto no supone ningún cambio sustancial en relación a las atenciones hacia Rosa las cuales siguen siendo de atención personal: baño completo, lavado de pelo semanalmente y masaje de piernas con crema hidratante; y atención doméstica: limpieza básica de la vivienda. Pasadas ocho semanas el Instituto Murciano de Acción Social resuelve grado II de dependencia lo que posibilita el trámite de solicitud de plaza residencial. Se realiza visita domiciliaria para poner en conocimiento de Rosa que ya es posible formalizar la solicitud de plaza residencial, Rosa reitera su voluntad de irse a una residencia pues según refiere:

"La relación entre mi hija y yo es cada vez más conflictiva" (Ent 4.)

Se le informa que pasará un año hasta la concesión de plaza residencial.

Por su lado Dori manifiesta su aceptación a ir a un Centro de Día, pero también la imposibilidad de acudir pues tiene que hacerse cargo de su madre y de su sobrino.

Desde el servicio de ayuda a domicilio se realizan innumerables gestiones telefónicas a la hija menor Miriam (madre del menor) la cual figura como miembro de contacto para cuestiones administrativas y/o burocráticas. El contacto se hace imposible, no acude a las citaciones y tampoco atiende las llamadas.

Desde el servicio de ayuda a domicilio se realizan reuniones semanales de supervisión de casos con todo el equipo de auxiliares, y durante la reunión es cuando se recoge el testimonio de la auxiliar de hogar que acude al domicilio de Rosa y en esta ocasión declara:

"Que las relaciones son muy conflictivas a nivel verbal por ambas partes, Rosa recibe cocotazos de su hija Dori cuando expulsa gases según refiere Rosa, y que en los últimos días al realizar el aseo íntimo de Rosa visualizo hematomas en los brazos".

Se establece coordinación con el Centro de Salud, al tiempo que se recibe aviso de emergencia por parte de la trabajadora social del mismo sobre la situación de riesgo detectada tras una visita de reconocimiento de la médico de familia, donde han obtenido testimonio de Rosa de estar recibiendo golpes por parte de su hija, sentir temor por su integridad física y manifiesta su deseo de salir del domicilio. La doctora emite informe de detección de hematomas y otros indicadores, el cual fue clave para poder proceder con carácter urgente hacia las dos vías posibilitadoras de protección del sistema, dada la situación de indefensión de ambas convivientes dependientes.

Desde el servicio de ayuda a domicilio se activa el procedimiento de solicitud de plaza residencial por emergencia social al Instituto Murciano de Acción Social con un informe detallado, emitido y firmado por la trabajadora social del servicio y del Centro de Salud. En apenas dos semanas este comunica la concesión de plaza por emergencia social, comunicándoselo a Rosa con la mayor premura. Tres días después se presenta sin cita previa en Servicios Sociales municipales $\mathrm{M}^{\mathrm{a}}$ Carmen, nuera de Rosa y esposa de su hijo Arturo, para exponer en tono ofensivo dos mensajes contradictorios, por un lado sus quejas por haber realizado dichos trámites sin informar a todos los hijos y por otro clarificar que no habrá compromiso por su parte ni la de su esposo para hacerse cargo de los cuidados de Rosa ya que son conocedores no sólo de la situación sino de más detales delicados que Servicios Sociales desconocen.

Dos días después se personan también en esta ocasión sin cita previa Alfonso, el marido de Rosa, y su hija menor Miriam, la cual expone que su madre renuncia a la plaza residencial voluntariamente (aporta renuncia firmada en el mismo día) y que ella se hará cargo de sus cuidados. Se le devuelve la imposibilidad de contar con ella a lo largo de la intervención, no ha respondido a las llamadas, ni mensajes de voz, ni citaciones oficiales... y las actuaciones responden en todo momento a las demandas de Rosa, persona con plenas facultades cognitivas. Miriam responde con actitud agresiva 
siendo el padre quien intenta apaciguarla. Alfonso el marido manifiesta:

"Mi esposa no ingresará en residencia pues somos una familia unida y un matrimonio que se quiere, yo volveré al domicilio familiar y me haré cargo de mi esposa y de mi hija Dori, en cuanto a la existencia de orden de alejamiento fue un error administrativo, todo está solucionado".

Desde el punto de vista jurídico, María Fernanda Moretón (2011) señala el reconocimiento de derechos de personas en situación vulnerable en la llamada Ley de Dependencia, y pone el ejemplo del derecho a no ser internados sin consentimiento. Sin embargo, dentro del campo de actuación del Sistema de Servicios Sociales es su competencia intervenir desde una doble función: la de control y también la de protección teniendo siempre en cuenta la decisión del sujeto en plenas facultades cognitivas como es el caso de Rosa, es por ello que se actúa poniendo en conocimiento del órgano superior competente (Ministerio Fiscal) la situación de desprotección en la que permanece Rosa con su renuncia de ingreso en residencia, la continuidad en el domicilio junto a su cuidadora e hija Dori, y ahora el retorno del esposo para asumir las funciones de cuidador de ambas. En suma se actúa protegiendo a Rosa mediante el instrumento controlador de denunciar ante el órgano competente (Fiscalía) la decisión tomada por Rosa.

Desde el servicio de ayuda a domicilio junto con la asesora legal de Servicios Sociales se convoca a todos los miembros de la unidad familiar, con la finalidad de elaborar un documento que recoja los compromisos de cada uno en relación a las atenciones hacia Rosa y Dori. Se les informa que dicho documento junto con el expediente completo será emitido a Fiscalía para que resuelva conforme a la situación del caso y los nuevos compromisos familiares, así como también la valoración profesional de continuidad o agravamiento de la situación de desprotección de la persona mayor y su cuidadora.

A modo de resumen se reflejan los compromisos adquiridos por los miembros de la unidad familiar, en relación a las atenciones y cuidados que desempeñarán hacia Rosa y Dori:

Alfonso, el marido se compromete a trasladarse de nuevo al domicilio familiar para atender a su esposa. Miriam, la hija menor manifiesta que se hará cargo de los cuidados hacia su madre y su hermana en la franja horaria que no esté trabajando, y en relación a su hijo niega en todo momento que hubiera sido atendido por su hermana Dori. El resto de her- manos manifiestan que acudirán al domicilio familiar en función de sus posibilidades. Finalmente expresan la necesidad de continuidad del Servicio de Ayuda a Domicilio, estableciendo un servicio privado hasta que dicho servicio público pueda de nuevo restablecerse.

Desde Servicios Sociales de Atención Primaria se emite a Fiscalía el expediente completo: informe social, informe de factores de riesgo y desprotección, informe médico, compromisos adquiridos por la unidad familiar en relación a los cuidados hacia Rosa y Dori tras la renuncia del recurso de residencia, así como también se adjunta la valoración y propuesta a Fiscalía por parte de los profesionales que han intervenido en el caso de cara al estudio del expediente por el órgano judicial competente.

Dicha valoración y propuesta expone lo siguiente: 1) Una vez descrita y reflejada la situación de desprotección detectada por acción u omisión hacia la persona mayor dependiente Rosa, por parte de su hija Dori, así como también los factores de riesgo detectados en Dori como consecuencia del desempeño del rol de cuidadora principal de dos personas incapaces: una persona mayor dependiente y un menor. Presentando ella a su vez limitaciones severas que imposibilitan el propio autocuidado, y siendo conocedores y consentidores en el tiempo de dicha situación el resto de miembros de la familia que no conviven en el domicilio. 2) Descritas las actuaciones profesionales realizadas por parte de Servicios Sociales de Atención Primaria y centro de salud, todas ellas bajo la demanda de Rosa en plenas facultades cognitivas, y finalmente la renuncia de la usuaria al ingreso en residencia, quedando la situación en un continuum de desprotección. 3) Recogidos los compromisos de la unidad familiar adquiridos en relación a los cuidados de Rosa y Dori. 4) Se presenta la valoración y propuesta profesional del caso, en la que los compromisos adquiridos son insuficientes, equivalentes a la situación actual e incluso pueden suponer un aumento en los factores de riesgo, por ello se propone:

- Estudio del expediente de la situación de desprotección de Rosa y Dori por parte de Fiscalía.

- Valoración de posibles medidas cautelares que protejan a la persona mayor, con posible ingreso en residencia de Rosa por no estar garantizados los cuidados e integridad física de esta.

- Valoración de la situación de desamparo en la que quedaría Dori a consecuencia del ingreso en residencia de su madre, y por ello se propone también el ingreso de Dori en un centro específico y próximo al de Rosa. 


\section{DESENLACE DEL CASO}

Con fecha octubre de 2016 la unidad familiar es citada por el órgano judicial competente, durante la vista los miembros reiteran los acuerdos en relación a los cuidados que se comprometen a ejercer hacia Rosa y Dori. De esta manera el expediente concluye con la devolución a los Servicios Sociales municipales para la continuidad del seguimiento y restablecimiento del servicio de ayuda a domicilio.

Desde octubre de 2016 hasta la actualidad la situación socio-familiar y relacional de la unidad de convivencia formada por Rosa, Alfonso y Dori se desarrolla con total normalidad, sin incidencias destacadas. La organización familiar que se estableció y hasta la fecha no ha variado y es la siguiente:

Rosa permanece en el domicilio según su voluntad junto a su esposo Alfonso, cuya vuelta al hogar fue aceptada y acordada por todos los miembros de la unidad familiar. Las atenciones personales y domésticas necesarias para el buen estado de salud son llevadas a cabo por Alfonso que hace las funciones de supervisión y/o atención en los momentos que no hay servicios, la hija menor Miriam acude por las tardes y fines de semana y cuentan con dos servicios: ayuda a domicilio realizado por la misma auxiliar de hogar, la cual hace de testigo de la estabilidad y buen funcionamiento familiar; también cuentan con un servicio privado el cual hace de respiro familiar cuando así lo estiman. Es muy importante destacar que el estado de salud de Rosa no ha empeorado, sino que se ha producido cierta estabilidad e incluso recuperación en la movilidad y autonomía que se reflejan en las actividades básicas de la vida diaria como el aseo íntimo y la manipulación de objetos.

Por parte de Alfonso, tras su vuelta al domicilio familiar, no se han conocido episodios de violencia de género y la convivencia se desarrolla con total normalidad.

En cuanto a Dori se destaca que el movimiento producido en la dinámica familiar, le permitió no solo liberarse del papel de cuidadora principal sino además la expresa manifestación de acudir a un Centro de Día. Se resalta que a día de hoy continúa asistiendo puntualmente al Centro de Día, siendo evidente su mejoría en cuanto a pérdida de peso, relaciones con el exterior, control en la medicación, etc.

Finalmente a excepción de Miriam, la hija menor, el resto de hijos hacen sus vidas de manera independiente y apenas existe relación, de igual modo que en tiempos anteriores.

\section{REFLEXIONES SOBRE LOS CONCEPTOS SENSIBILIZANTES}

Sócrates utilizó la mayéutica como método a través del cual el maestro hacía que el alumno, por medio de preguntas, descubriera conocimiento, el meollo era siempre cuestionar, preguntar, revisar, reflexionar y descubrir.

El presente artículo a través de la fenomenología descrita en la historia social de un caso de persona mayor y su cuidadora, ambas en situación de desprotección, ha desempeñado la función de la maestra que lleva, invita, induce sutilmente al alumno, que en este caso ha sido la profesional, a plantear dos objetivos que emergen del propio proceso y desarrollo de la intervención, los cuales hemos denominado conceptos sensibilizantes:

1. Existen dos tiempos krhonos y kairós que marcan una intervención social, ambos tiempos se desarrollan de manera desacompasada generando movimientos, comportamientos y actuaciones en direcciones diferentes y distantes. El tiempo khronos, es el tiempo analógico que enmarca la intervención profesional bajo el paraguas de la institución, los protocolos y con el idóneo objetivo y meta que alcanzar de proteger a las personas o sujetos en situación de desprotección, existe por tanto un tiempo y un espacio delimitado en la actuación y quehacer profesional. Sin embargo bajo la misma intervención también se da un tiempo kairós en los sujetos protagonistas de la intervención, se trata de un lapso de tiempo indeterminado en el que algo importante sucede y es ahí donde se produce el cambio y/o transformación de la situación o problemática de la historia del caso.

2. De ahí la historia social del caso como argumento privilegiado que proporciona la inevitable y necesaria revisión y autorreflexión de la praxis profesional. Se trata pues de una primera prueba de fuego para según la retroalimentación por parte de los/as colegas lectores, comenzar la responsable tarea reflexiva de acceso a la realidad siempre problemática y subjetiva de los casos y todo ello con el tiempo kairós como brújula.

Pareciera que el inesperado desenlace del caso pusiera en jaque el quehacer profesional, pero pasados los años ha resultado que tanto en su momento (octubre de 2016) como años después se pude desprender que sin el maremágnum de actuaciones no se hubieran producido los movimientos sistémicos en la unidad familiar, eso parece claro, sin embargo, no nos sirva de consuelo. La sensación interna pasado el tiempo kairós y khronos parece haberse acompasado llegando a una meditación 
madura y serena repleta de cuestionamientos: ¿Se actuó bajo la inercia de demanda-respuesta? ¿Se actuó bajo la urgencia de la protección como único objetivo? ¿Quizá la estructura, organización y composición de funcionamiento del servicio de ayuda a domicilio, tanto a nivel municipal como por dependencia, no estaba ni está dotado para responder a la problemática realidad de los casos de personas mayores y de salud mental? ¿Existe algún programa específico que lleve a cabo dichas intervenciones de manera biopsicosocial? ¿El sistema lo proporciona? O ¿Dónde estaba la clave? Quizá en haber podido diferenciar entre la verdad emocional de Rosa, sensible y subjetiva, y la verdad objetiva de las actuaciones y marcos competenciales de los Servicios Sociales. Para esto último y de manera contundente hacía falta tiempo khronos y sobre todo el tiempo kairós propio de un caso en proceso, imprescindible para poder llegar a una toma de conciencia de la problemática real.

De cualquier modo la realidad aquí y ahora es que los/ as profesionales de la intervención social, penetramos en la difícil realidad de los sujetos de intervención intentando geometrizarla y hacerla objetiva, buscando la estructura del caso sea con los medios que sea, recordando la idea de que el profesional es el principal instrumento y de esta manera nos olvidamos y nos alejamos de la complejidad profunda del fondo, quedándonos en la figura o superficie sensible y aparente. La práctica usual sobrepasada por la urgencia, hace frecuente que nos quedemos en la superficie, en la corteza del fenómeno, reduciendo así su pluridimensionalidad.

Con todo ello con el presente trabajo se comparte la praxis profesional y la autorreflexión posteriori, la cual no solo ha sido una lección profesional y de vida, sino de la que invito a los profesionales y cualquier alumno mayéutico a la tarea responsable de revisar y cuestionar la práctica profesional del trabajo social.

El leitmotiv de nuestra tarea como trabajadores sociales no es otra que la duda metódica con el propósito de poder interpretar fenómenos complejos en su contexto adecuado. Necesitamos pues de kairós para la reflexión, para el darse cuenta y para la toma de conciencia de nuestro trabajo y esa es nuestra responsabilidad social.

\section{CONCLUSIONES FINALES}

Los profesionales del trabajo social deberíamos mantener siempre abierta la dialéctica de lo que es el trabajo social, el ser y quehacer.

La disciplina del trabajo social implica alcanzar un cambio pero no siempre va en la dirección marcada como meta en la intervención.
No funciona la idea romántica de agente de cambio si esta implica la pérdida de la claridad de las subjetividades implicadas que son las que realmente permiten contribuir al cambio.

Debemos reflexionar sobre la idea mesiánica de la profesión.

El cambio se opera desde el ámbito de la disfuncionalidad y al final se trata de superar la situación deficiente y no ha de ser por el camino pautado.

El discurso de la profesión que lleva al "yo creo..." deja y muestra inseguridad en la profesión y en el concepto de trabajo social, ahora bien, también nos hace agentes pasivos de la reproducción de las diferentes formas de actuación cristalizadas en las instituciones.

Sin más pretensión de crítica, el presente trabajo pone la intención en el objetivo de la revisión y autorreflexión de la práctica profesional en las intervenciones de casos cuyo desenlace se resuelve muy equidistante al marcado.

A veces nos ponernos al servicio de la política pública y los protocolos, olvidándonos de generar reflexiones sobre los efectos que las intervenciones tienen en las personas con las que trabajamos.

La práctica concreta se confronta con la realidad subjetiva del sujeto.

La praxis no se puede encorsetar, sírvanos la historia social de un caso, las historias de vida para revisarnos y reflexionar con el tiempo kairós al timón.

Es el sujeto quien marca el tiempo de la intervención y quien da la mirada de por dónde seguir. Lo primero siempre es el sujeto.

\section{REFERENCIAS BIBLIOGRÁFICAS}

Bazo, M. T. (2003). Violencia familiar contra las personas ancianas que sufren dependencia y enfermedad. Española de Geriatría y Gerontología, 36(1), 8-14.

Bourdieu, P. (1999). La Miseria del mundo, Madrid: Akal.

Chárriez, M. (2012). Historias de vida: Una metodología de investigación cualitativa. Griot, 5(1), 50-67.

Cooney, C. \& Mprtimer, A. (1995): Elder abuse and dementia: A pilot study, International Journal of Social Psychiatry, 4(4), 276-283.

Ferrarotti, F. (2007). Las historias de vida como método. Convergencia, 44, 15-40.

Ley 3/2003 de 10 de abril del Sistema de Servicios Sociales de la Región de Murcia. Boletín Oficial de la Región de Murcia n99, de 2 de mayo de 2003.

Ley 39/2006, de 14 de diciembre, de Promoción de la Autonomía Personal y Atención a las Personas en Situación de 
Dependencia. BOE n 299, de 15 de diciembre de 2006. Madrid, España. Recuperado el 13 de agosto de 2018 de https://www.boe.es/eli/es/l/2006/12/14/39/con

Moretón, M.F. (2011). La inacabada reforma psiquiátrica española: cuestiones sobre internamientos psiquiátricos no voluntarios y la ausencia de control judicial en los ingresos geriátricos. Comunitania, 1, 69-87.

Moya Bernal, A y Barbero Gutiérrez, J. (2005). Malos tratos a personas mayores: Guía de actuación. Madrid: IMSERSO.

Muñoz, J. (2004). Personas mayores y malos tratos. Madrid: Pirámide.

Navarro, V. (2016). Perfil de los cuidadores informales y ámbito de actuación del trabajo social. Trabajo Social Hoy, 77,6384.

Pastor, E. y Sánchez, M (2013). Impacto de la crisis económica y los cambios en las políticas sociales en el perfil, necesidades y prestaciones de las personas usuarias de los servicios sociales municipales. Análisis de caso local en la región de Murcia (España) Alternativas, 3, 141-161.

Quílez, A. (2016). Servicios sociales comunitarios ante un caso de persona mayor semiabandonada con demencia. Azarbe, 5, 32-35.
Rodríguez, C. (2014). Ética y Trabajo social: La reflexión de la profesión, camino de la ciudadanía. Azarbe, 3, 55-57.

Taylor y Bogdan (2000). Introducción a los métodos cualitativos. Barcelona: Paidós.

Touza, C. (2008). Personas Mayores en Riesgo, Detección del maltrato y la autonegligencia. Universidad de Islas Baleares: Pirámide.

Uriz, M.J., Ballesteros, A. Viscamet, J.J. (2013). Funciones profesionales de los Trabajadores sociales en España. Cuadernos de trabajo social, 26(1), 127 - 138

Vázquez, O. (2013). Investigar para fortalecer la dimensión política del Trabajo social. En VI Jornadas de trabajo social. Universidad País Vasco. Investigación y trabajo social, 51 - 64.

Vigo, A. (2015). Hans - Georg Gadamer y la filosofía hermenéutica: la comprensión cómo ideal y tarea. Estudios Públicos, 87.

Viscarret, J.J (2002). Modelos y métodos de intervención en Trabajo Social. Madrid: Alianza.

Zambrano, I., Martín, M. T., Muñoz, J. M., Olid, E. (2015). La organización social de los cuidados de larga duración en un contexto de austeridad y precariedad. Zerbitzuan, 60, 113-130. 